\title{
Are FXR Family Proteins Integrators of Dopamine Signaling and Glutamatergic Neurotransmission in Mental Illnesses?
}

\author{
Jivan Khlghatyan ${ }^{1,2}$ and Jean-Martin Beaulieu ${ }^{1,2 *}$ \\ 'Department of Pharmacology and Toxicology, Medical Sciences Building, University of Toronto, Toronto, ON, Canada, \\ ${ }^{2}$ Department of Psychiatry and Neuroscience, Faculty of Medicine, Université Laval, Quebec, QC, Canada
}

OPEN ACCESS

Edited by:

Fang Liu,

Centre for Addiction and Mental

Health, Canada

Reviewed by:

Fabio Fumagalli,

Università degli Studi di Milano, Italy

Raul Gainetdinov,

Fondazione Istituto Italiano di

Tecnologia, Italy

*Correspondence:

Jean-Martin Beaulieu

martin.beaulieu@utoronto.ca

Received: 01 April 2018

Accepted: 29 June 2018

Published: 24 July 2018

Citation:

Khlghatyan J and Beaulieu J-M

(2018) Are FXR Family Proteins

Integrators of Dopamine Signaling and Glutamatergic Neurotransmission

in Mental Illnesses?

Front. Synaptic Neurosci. 10:22.

doi: 10.3389/fnsyn.2018.00022
Dopamine receptors and related signaling pathways have long been implicated in pathophysiology and treatment of mental illnesses, including schizophrenia and bipolar disorder. Dopamine signaling may impact neuronal activity by modulation of glutamate neurotransmission. Recent evidence indicates a direct and/or indirect involvement of fragile $X$-related family proteins (FXR) in the regulation and mediation of dopamine receptor functions. FXRs consists of fragile $X$ mental retardation protein 1 (Fmr1/FMRP) and its autosomal homologs Fxr1 and Fxr2. These RNA-binding proteins are enriched in the brain. Loss of function mutation in human FMR1 is the major genetic contributor to Fragile X mental retardation syndrome. Therefore, the role of FXR proteins has mostly been studied in the context of autism spectrum disorders. However, recent genomewide association studies have linked this family to schizophrenia, bipolar disorders, and mood regulation pointing toward a broader involvement in mental illnesses. FXR family proteins play an important role in the regulation of glutamate-mediated neuronal activity and plasticity. Here, we discuss the brain-specific functions of FXR family proteins by focusing on the regulation of dopamine receptor functions, ionotropic glutamate receptors-mediated synaptic plasticity and contribution to mental illnesses. Based on recent evidence, we propose that FXR proteins are potential integrators of dopamine signaling and ionotropic glutamate transmission.

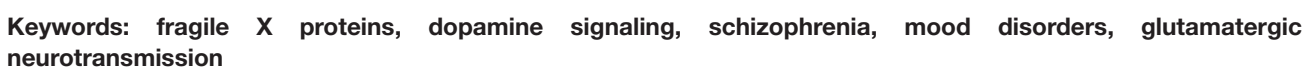

\section{INTRODUCTION}

The neurotransmitter dopamine is involved in the regulation of several behavioral dimensions including locomotion, reward, affect, mood, and cognitive functions. Dopamine exerts its actions by activating two groups of $\mathrm{G}$ protein couple receptors termed D1-class (D1 and D5) and D2-class (D2S, D2L, D3, and D4) (Missale et al., 1998; Beaulieu and Gainetdinov, 2011). D1-class receptors are positively coupled to adenylate cyclase to stimulate the production of the second messenger cAMP. In contrast, D2-class receptors inhibit cAMP production. The D2 dopamine receptor (D2R) also exerts its actions by engaging a beta-arrestin 2 ( $\beta$ Arr2) mediated signaling pathway resulting in the inactivation of the protein kinase Akt and the concomitant activation of its substrates glycogen synthase kinase 3 alpha and beta (Gsk3 $\alpha / \beta$ ) (Beaulieu et al., 2004, 2005).

One major downstream consequence of dopamine receptor signaling is the regulation of ion channels and ionotropic receptors for neurotransmitters (Snyder et al., 2000; 
Svenningsson et al., 2004; Beaulieu and Gainetdinov, 2011; Devor et al., 2017). A great deal of attention has been given to the roles of cAMP and protein kinase A-mediated signaling in the regulation of ionotropic glutamate receptors $\alpha$-amino-3-hydroxy-5-methyl4 -isoxazole propionic acid (AMPA) and $\mathrm{N}$-methyl-D-aspartate (NMDA). Furthermore, dopamine receptor signaling has also been shown to regulate the expression of genes involved in ionotropic glutamate transmission (Yao et al., 2004; Renthal and Nestler, 2008; Renthal et al., 2008).

The fragile $\mathrm{X}$ mental retardation syndrome-related protein 1 (Fxr1), Fxr2, and fragile $\mathrm{X}$ mental retardation protein 1 (Fmr1) comprise a small family of RNA binding proteins named fragile X-related (FXR) proteins. Mutations in Fmr1 gene are linked to human fragile $\mathrm{X}$ syndrome and autism (Crawford et al., 2001). However, recent genetic studies linked FXR family to schizophrenia, bipolar disorders, and mood regulation, indicating their wider role in adult-onset mental disorders (Bowden et al., 2008; Fromer et al., 2014; Purcell et al., 2014; Schizophrenia Working Group of the Psychiatric Genomics Consortium, 2014; Takata et al., 2017). Dopamine-mediated signaling is impaired in many of these pathologies (Howes and Kapur, 2009; Ashok et al., 2017). In addition, recent studies point toward possible direct or indirect implications of FXR family proteins in dopamine receptor regulation and signaling. Here, we explore the possible contribution of FXR proteins to the integration of dopamine and ionotropic receptor-mediated glutamate signaling in the broader context of adult onset mental disorders and addiction.

\section{FXR PROTEINS IN THE BRAIN}

The FXR family proteins are enriched in the brain. Immunolabeling revealed widespread expression of Fxr1, Fxr2, and Fmr1 in fetal and adult human and mouse brains. These proteins are mostly localized to the cytoplasm and proximal dendrites of neurons. In addition, the Fxr1 expression is also found in the nucleus of neurons in the fetal human brain. Very limited expression of Fxr1 was observed in glia by immunostaining (Tamanini et al., 1997; Bakker et al., 2000; Cook et al., 2011). However, recent investigations using RNA-Seq identified transcripts of Fxr1, Fxr2, and Fmr1 in different cell types of the mouse cortex. In contrast to the Fmrl transcript, which is mostly present in neurons and astrocytes, Fxrl and Fxr2 transcripts have also been detected in oligodendrocytes, microglia, and endothelial cells in the cortex of mice (Thomsen et al., 2013; Zhang et al., 2014).

Investigation of subcellular localization by immunoelectron microscopy and biochemical methods showed that all three FXR proteins are associated with polyribosomes in the cytoplasm and along the dendrites of neurons (Tamanini et al., 1997; Bakker et al., 2000; Darnell et al., 2009; Cook et al., 2011). All FXRs are detected in mouse brain synaptosomes (Tamanini et al., 1997; Cook et al., 2011). Fxr1 and Fmr1 are observed in the base of dendritic filopodia and spines in proximal and distal dendrites of hippocampal cultures (Antar et al., 2005; Cook et al., 2011). Subsets of dendritic Fxr1 clusters also contain Fxr2 and
Fmr1 (Cook et al., 2011). Fxr1, Fxr2, and Fmr1 are enriched in RNA granules (stalled ribosomes) in the developing mouse brain. Interestingly, all FXR proteins are co-detected only in $22 \%$ of RNA granules, the rest of the granules have different combinations of two or only one FXR protein, indicating possible overlapping and nonoverlapping functions of FXR proteins in RNA granules (El Fatimy et al., 2016).

The FXR family proteins are also found in presynaptic compartments in so-called presynaptic fragile X granules (FXGs). Despite the presence of FXR proteins in the somatodendritic compartment of virtually every neuron, their presynaptic localization is more restricted. FXGs are detected in a subset of neurons in frontal cortical, hippocampal area CA3, and olfactory bulb glomeruli, only in developing mouse brains. Studies of granule composition showed that all three FXR family proteins were present in cortical FXGs, while the majority of granules in the hippocampus and olfactory glomeruli only contained Fxr2 and Fmr1. Knockout mouse studies demonstrated that Fxr2 is crucial for formation and Fmrl for regulation of FXGs (Christie et al., 2009). Overall, expression and subcellular localization data suggest that FXR proteins can have shares as well as exclusive brain functions.

\section{FXR PROTEINS ARE REGULATORS OF IONOTROPIC GLUTAMATE RECEPTOR-MEDIATED SYNAPTIC PLASTICITY}

The contribution of Fmr1 and Fxr2 to the regulation of metabotropic glutamate receptor functions has been studied extensively. For example, Fxr2 KO mice exhibit a reduction in metabotropic glutamate receptor-dependent long-term depression (mGluR-LTD), while the same type of plasticity is enhanced in Fmr1 and Fxr2/Fmr1 double knockout mice. Further characterization of underlying mechanisms pointed toward a dependence of mGluR-LTD on protein synthesis in Fxr2 KO mice, while it would be only partially dependent upon protein synthesis in Fxr2/Fmrl double KO mice (Zhang et al., 2009).

However, there is also evidence for an implication of these proteins in the regulation of ionotropic glutamate receptor functions. Fxr2 binds to the GluA1 mRNA coding sequence, stabilizing this transcript and regulating GluA1 expression. In line with this, Fxr2 also affected GluA1-mediated currents and surface availability. Fmr1 deficiency also results in decreased GluA1. However, this effect would not be mediated by a regulation of GluA1 synthesis, but a contribution of Fmr1 to GluA1 membrane expression (Figure 1). From a functional point of view, young mice lacking Fmr1 or both Fxr2 and Fmr1 display altered basal transmission, paired-pulse facilitation, and protein synthesis-dependent late-phase LTP (L-LTP). However, these parameters are unaffected at all ages in Fxr2 KO mice.

The neuronal functions and the regulation of glutamate neurotransmission by Fxr1 have been understudied as compared to other FXR proteins. Conditional knockout (cKO) of Fxr1 


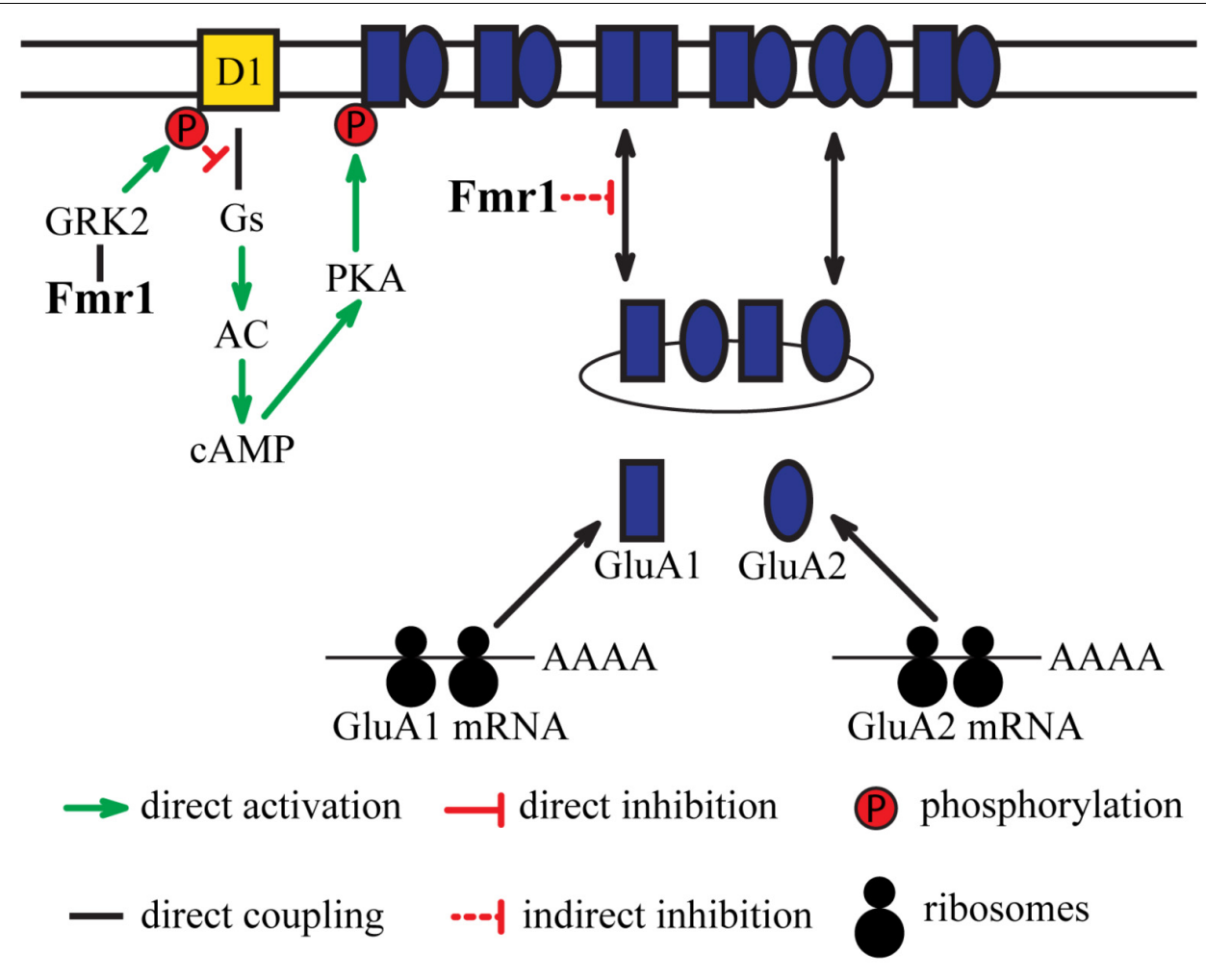

$\uparrow$ membrane insertion 2050 endosomes

FIGURE 1 | Integration of dopamine receptor D1-mediated signaling and glutamatergic neurotransmission by Fmr1. D1 receptor-mediated signaling results in phosphorylation of GluA1 subunits of AMPA receptors. Fmr1 regulates phosphorylation of D1 receptors via direct interaction with GRK2. This regulation of D1 by Fmr1 subsequently impacts phosphorylation of GluA1. Fmr1 also regulates trafficking of GluA1 via unknown mechanism.

specifically from excitatory neurons of the forebrain resulted in a slight decrease in density and length of spines in hippocampal area CA1 cells (Cook et al., 2014). While normal basal synaptic strength, short-term plasticity, and protein synthesisindependent early phase long-term plasticity (E-LTP) were unaltered. However, Fxr1 cKO mice exhibit a significant increase in L-LTP compared to controls. This effect would be mostly due to an increase in AMPA receptor subunit GluA2, while NMDA receptors would be unaffected. Further analysis suggested that Fxr1 can directly regulate GluA2 by binding to a GU-rich element in the $5^{\prime}$ untranslated region (UTR) of GluA2 mRNA (Cook et al., 2014).

In contrast, adeno-associated virus (AAV)-mediated augmentation of Fxr1 levels in the adult medial prefrontal cortex (mPFC) resulted in a decrease of excitatory postsynaptic currents (EPSCs) amplitude and frequency. A similar effect was observed as a result of a CRISPR/Cas9-mediated reduction of Gsk3 $\beta$ expression in mPFC (Khlghatyan et al., 2018). Gsk3 $\beta$ can directly phosphorylate Fxr1 and negatively regulate its expression (Del'Guidice et al., 2015). Thus, modulation of EPSCs by Fxr1 can occur in response to its regulation by Gsk3 $\beta$. Further investigations revealed a decrease in synaptic AMPA receptors along with a possible switch from predominantly heteromeric
GluA1/GluA2 containing to homomeric GluA1 AMPA receptors (Khlghatyan et al., 2018).

\section{A ROLE FOR Fmr1 IN DOPAMINE D1 RECEPTOR SIGNALING AND REGULATION}

Evidence exists for the functional interplay between the D1 receptor and Fmr1. Stimulation of D1 has been shown to cause dynamic changes in phosphorylation of Fmr1 and result in a regulation of translation of its target proteins (Wang et al., 2010). Stimulation of these receptors by agonists also increases surface expression of the GluA1 subunit. The effect of D1 agonists on surface GluA1 expression is abolished in Fmr1 KO mice. Additionally, electrophysiological recordings of mEPSCs showed that Fmr1 is necessary for D1 receptor-mediated synaptic integration of GluA1 subunit. Moreover, D1 receptor-mediated facilitation of LTP in the cingulate cortex is altered in Fmr1 $\mathrm{KO}$ mice, indicating the contribution of Fmr1 in D1 receptormediated synaptic plasticity (Figure 1).

Further studies revealed that D1 receptor is also hyperphosphorylated in the PFC of Fmr1 KO mice, which 
results in impaired coupling to Gas proteins. G proteincoupled receptor kinases (GRKs) are known to phosphorylate dopamine receptors and regulate their binding to $G$ proteins and beta-arrestins. Two classes of GRKs, GRK2 class (GRK2 and GRK3), and GRK4 class (GRK5 and GRK6) have been shown to differentially regulate D1 and D2 receptors (Gurevich et al., 2016). The absence of Fmr1 caused a redistribution of GRK2 to the surface that resulted in hyperphosphorylation of D1 receptors by GRK2 (Figure 1). No changes in GRK4 localization were observed in Fmr1 KO mice (Wang et al., 2008). However, it is possible that interaction between other FXR proteins and GRKs may exist and affect dopamine receptor functions. Notably, alterations of GRK6 levels have been shown to specifically affect D2 receptors mediated signaling (Gainetdinov et al., 2003; Gurevich et al., 2016). It may thus be of interest to investigate if an alteration in FXR proteins functions may also affect GRK6 and D2 mediated signaling.

\section{DOES FXr1 PLAY A ROLE IN DOPAMINE D2 RECEPTOR BETA-ARRESTIN-2-MEDIATED SIGNALING?}

New evidence also suggests that Fxr1 may play a role in the regulation of ionotropic glutamate receptors by the D2 dopamine receptor. Dopamine regulates Gsk3 $\beta$ activity downstream of D2 (Beaulieu et al., 2004, 2005). Activation of D2 results in the in dephosphorylation and inactivation of Akt following the formation of a protein complex comprised of $\beta$-Arrestin-2 ( $\beta$ Arr2), protein phosphatase $2 \mathrm{~A}$ (PP2A) and Akt. This, inhibition of Akt by the D2 receptor prevents the inhibitory phosphorylation of Gsk3 $\beta$ by Akt and results in Gsk3 $\beta$ activation (Beaulieu et al., 2005). The $\beta$ Arr2/PP2A/Akt complex can be disrupted by lithium, resulting in an activation of Akt and subsequent phosphorylation and inactivation of Gsk3 $\beta$ (Beaulieu et al., 2008).

In addition to lithium, other mood stabilizers, such as lamotrigine and valproate can also indirectly inhibit brain Gsk3 $\beta$. This inhibitory effect is absent in D2 $\mathrm{KO}$ and $\beta$ Arr2 $\mathrm{KO}$ mice, indicating possible engagement of D2R- $\beta$ Arr2-PP2AAkt pathway (Del' Guidice and Beaulieu, 2015). Chronic treatment with lithium, lamotrigine, and valproate increases Fxr1 expression in the striatum and prefrontal cortex. An effect that is absent in $\beta$ Arr2 KO mice (Del'Guidice et al., 2015) thus suggesting an engagement of D2R- $\beta$ Arr2-Gsk3 pathway in the regulation of Fxr1 levels by these drugs.

Reduction of Gsk3 $\beta$ and augmentation of Fxr1 in mPFC caused a decrease in synaptic GluA1 and GluA2 subunits of AMPA receptors without affecting their synaptosomal expression levels. This indicates that the Gsk3 $\beta$-Fxr1 pathway may be involved in regulation of the synaptic delivery of GluA1 and GluA2 (Figure 2) (Khlghatyan et al., 2018). Interestingly, chronic treatment with lithium or valproate reduces synaptosomal GluA1 levels and surface GluA1 distribution (Du et al., 2004). This effect is similar to what was observed following augmentation of Fxr1 or reduction of Gsk3 $\beta$ levels in the mouse mPFC (Khlghatyan et al., 2018). This suggests a contribution of the $\beta$ Arr2-Gsk3-Fxr1 pathway in the effect of lithium and valproate on surface GluA1 (Figure 2). A regulation of Fxr1 by $\beta$ Arr2-mediated D2 signaling would also provide a mechanism by which this receptor may regulate local protein synthesis and AMPA neurotransmission. However, while these observations are tantalizing the direct regulation of Fxr1 and GluA1 by D2 receptors still has to be further established by more investigations.

\section{FXR PROTEINS IN REGULATION OF DOPAMINE-ASSOCIATED BEHAVIORS}

Direct evidence for the contribution of dopamine in FXR proteins-mediated behaviors or the role of FXR proteins in dopamine-related behaviors remains scarce. However, there is evidence for an involvement of FXR proteins in the regulation of several behavioral modalities such as locomotion, gait, and sensory-motor gating, which are also regulated by dopamine neurotransmission.

Fxr2 KO mice exhibit hyperactivity in the open-field test, abnormal sensorimotor gating (acoustic startle response), impaired motor coordination, decreased response to heat stimulus, and impaired learning and memory performance in both contextual fear conditioning and the Morris water tasks (Bontekoe et al., 2002; Guo et al., 2015). Interestingly, Fmr1 KO mice exhibited several behavioral phenotypes similar to those of Fxr2 KO, such as hyperactivity and acoustic startle response impairment (Bontekoe et al., 2002; Spencer et al., 2006). Furthermore, some phenotypes like hyperactivity, sensory motor gating deficits, and fear conditioning are more pronounced in Fxr2/Fmr1 KO mice, indicating possible additive effects in specific behavioral paradigms (Bontekoe et al., 2002; Spencer et al., 2006). Interestingly, several behavioral phenotypes, such as hyperactivity of Fxr2 $\mathrm{KO}$ and Fmr1 $\mathrm{KO}$ mice and anxiety of Fmr1 KO mice were not replicated across studies (Spencer et al., 2006). A possible explanation for these discrepancies is that different genetic background and housing conditions can affect the penetrance of phenotypes associated to the deletion of these genes.

Stimulation of D1 receptor by agonists ameliorates hyperactivity phenotypes and motor coordination impairments in Fmr1 KO mice (Wang et al., 2008). Treatment with amphetamine also improved impaired novel object recognition in these mice (Ventura et al., 2004). In contrast, inhibition of Gsk $3 \beta$, which can be activated by $\mathrm{D} 2$ receptors, results in reduced hyperactivity, improved cognition, and social behavior in this same mouse model (Yuskaitis et al., 2010; Mines and Jope, 2011).

There is also evidence for a contribution of Fmr1 in the responsiveness to psychostimulant drugs, which exert their effects by increasing dopamine extracellular tones (Beaulieu and Gainetdinov, 2011). Expression of Fmr1 in the medial prefrontal cortex has been found to be reduced in adult rats that were exposed to cocaine during adolescence (Caffino et al., 2014) and a mutagenesis study identified the cytoplasmic FMRP interacting protein 2 as a modulator of acute and sensitized locomotor 

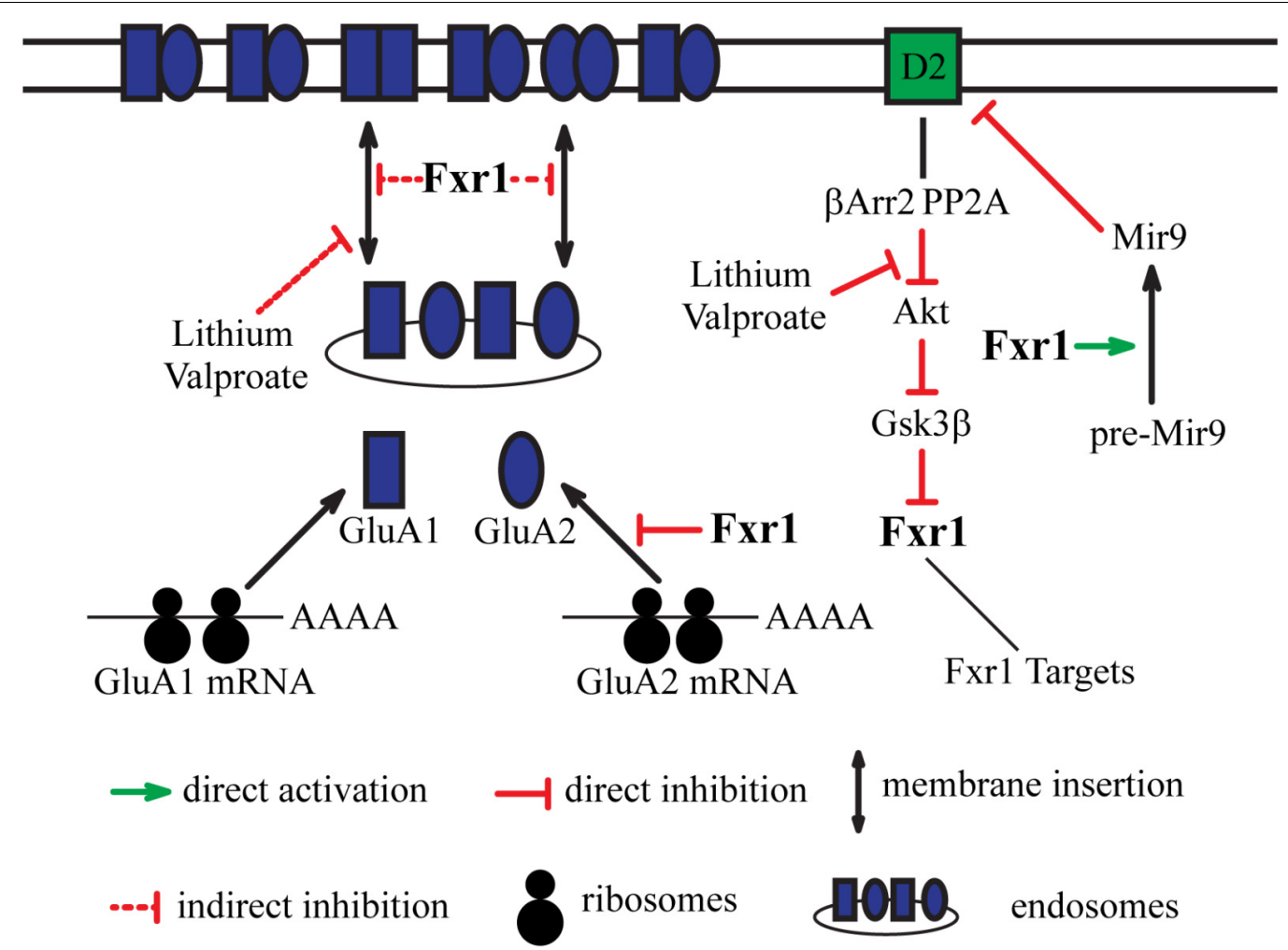

membrane insertion

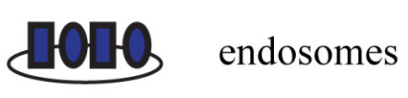

FIGURE 2 | Integration of dopamine receptor D2-mediated signaling and glutamatergic neurotransmission by Fxr1. Fxr1 regulates trafficking of GluA1, GluA2, and synthesis of GluA2 subunits of AMPA receptors. Lithium and valproate regulate Fxr1 levels downstream of D2- $\beta$ Arr2-Akt-Gsk3 $\beta$ pathway. Lithium and valproate also regulate trafficking of GluA1, suggesting possible mechanistic involvement of Fxr1. Fxr1 regulates biogenesis of schizophrenia related Mir9, which, in turn, negatively regulates translation of $\mathrm{D} 2$ receptors.

responses to both cocaine and methamphetamine (Kumar et al., 2013). In line with this, Fmr1 KO mice display altered behavioral sensitization and reward to cocaine (Smith et al., 2014). This effect appears to involve different functions of this protein in different brain regions. On the one hand, behavioral sensitization would require Fmrl expression in the adult nucleus accumbens, a region of the striatum that is involved in addiction-related behaviors. On the other hand, the regulation of mGluR 5 by Fmr1, possibly in multiple brain regions would explain the contribution of Fmr1 to cocaine-associated reward (Smith et al., 2014).

Fxr1 KO mice die shortly after birth from cardiac and respiratory failure, thus behavioral testing is only possible after brain-specific alterations of Fxr1 expression (Mientjes et al., 2004). Fxr1 cKO has been achieved in mouse forebrain pyramidal neurons using a CamKII-Cre system and Fxr1 floxed alleles (Cook et al., 2014). These mice show no deficits in basic motor/sensory function, anxiety, contextual fear memory, and working memory. However, forebrain Fxr1 cKO mice display enhanced ability to recall spatial memories in a modified Morris water maze paradigm. In addition, these mice fail to shift their preference to the new platform location during the reversal probe test, thus demonstrating altered behavioral flexibility.

In contrast to gene inactivation, Fxr1 AAV-mediated overexpression in $\mathrm{mPFC}$ neurons of adult mice affects anxietyrelated behaviors (Del'Guidice et al., 2015; Khlghatyan et al.,
2018). A similar behavioral signature was observed after reduction of Gsk3 $\beta$ levels in forebrain pyramidal neurons in Gsk3 $\beta$ cKO mice (Latapy et al., 2012) and as a result of more specific AAV-mediated gene inactivation in the mPFC (Del'Guidice et al., 2015; Khlghatyan et al., 2018). In line with this, the interaction between Fxr1 and Gsk3 $\beta$ has been shown to affect mood and emotional processing in healthy humans (Del'Guidice et al., 2015).

The difference between cKO of Fxr1 and region-specific viral vector-mediated manipulations points to a role for Fxr1 in the regulation of different behaviors in a brain region-specific manner. This also suggests that functional impact of alterations in Fxr1 levels may depend on developmental stage. Finally, development of new approaches to manipulate Fxr1 expression in specific populations of dopamine receptor expressing cortical neurons will be needed to further characterize the contribution of dopamine neurotransmission in these behavioral phenotypes.

\section{FXR PROTEINS, ADULT ONSET PSYCHIATRIC DISORDER ASSOCIATION}

Loss of function of Fmrl is one of the leading genetic causes of Fragile X and autism (Crawford et al., 2001). Structural similarities between FXR family proteins led to an investigation 
of Fxr1 and Fxr2 functions in the context of autism and fragile $\mathrm{X}$ syndrome. However, accumulation model of single nucleotide polymorphisms (SNPs) in FXR genes showed association with autistic traits not only in autistic patients but also in schizophrenics, patients with other neuropsychiatric disorders and healthy individuals, thus indicating a possible broader role of FXR genes (Stepniak et al., 2015). Indeed, recent human genetic and functional studies demonstrated the association of FXR genes and proteins to schizophrenia, bipolar disorders, and mood regulation.

Genome-wide association studies (GWAS) have linked SNPs in the FXR1 locus to schizophrenia (Schizophrenia Working Group of the Psychiatric Genomics Consortium, 2014). Later, a GWAS associated SNP in this locus was shown to be in linkage disequilibrium with splicing quantitative trait loci (sQTL) SNPs identified in the human dorsolateral prefrontal cortex (DLPFC). This suggests that a schizophrenia-associated SNP in FXR1 locus may possibly contribute to the disease by affecting alternative splicing (Takata et al., 2017). Mapping out potential schizophrenia networks by correlated expression of 369 genes from 108 schizophrenia-associated loci resulted in the identification of several pathways. The pathway with the largest number of correlations included 56 genes along with $D R D 2$ and FXR1 (Liedtke et al., 2017). Alterations in microRNAs also have been linked to schizophrenia. Interestingly, schizophreniarelated genes are more likely to be regulated by microRNAs and Mir9 targetome is the most enriched in schizophrenia risk genes. Fxr1 have been shown to regulate the processing of several brain-specific microRNAs, including Mir9 (Xu et al., 2011). In turn, Mir9 targets and negatively regulates $D R D 2$ transcripts via binding to their 3'UTR region (Shi et al., 2014; Hauberg et al., 2016). Additionally, the micro-RNA that is the most significantly associated to schizophrenia Mir137 has been shown to inhibit the expression of the Fxr1 negative regulator Gsk $3 \beta$ by acting on the $3^{\prime}$ UTR of the GSK3B mRNA (Thomas et al., 2017). This makes a strong case for the association of Fxr1 to schizophrenia and D2-mediated signaling (Figure 2). A possible contribution to the disease may be via alterations in splicing of Fxr1 with subsequent changes in the regulation of microRNAs and their targets such as the $\mathrm{D} 2$ receptor mRNAs.

Polymorphisms in the Fxr2 and Fmr1 loci have not been associated with schizophrenia by GWAS. However, some studies pointed to possible indirect contributions. Fxr2 levels have been shown to be altered in a small group of schizophrenia patient's brain (Bowden et al., 2008). Furthermore, genome-wide epigenetic analysis conducted in monozygotic twins identified significant changes in methylation profile of Fxr2 gene in schizophrenic patients compared to healthy subjects (Dempster et al., 2011). Several studies identified changes in Fmr1 protein levels in brains of schizophrenic patients (Fatemi and Folsom, 2011). Decreased levels of Fmr1 may be associated with lower IQ and earlier onset of the disease (Kovács et al., 2013). Furthermore, recent large-scale studies indicated enrichment of Fmrl targets in the genetic architecture of schizophrenia (Fromer et al., 2014; Purcell et al., 2014).

In addition to schizophrenia, some studies indicated that Fmrl targets are enriched within genes that are shown to be affected in bipolar patients (Folsom et al., 2015; Goes et al., 2016). Furthermore, GWAS studies have identified SNPs in FXR1 gene that shows significant association to bipolar disorder and comorbid eating disorder. Interestingly, anxiety spectrum disorders were co-occurring with a much higher rate in bipolar patients with an eating disorder (Liu et al., 2016). Thus, indicating the possible broad involvement of Fxr1 in the regulation of mood. Indeed, an interaction between functional polymorphisms in FXR1 and GSK3B contributes to mood regulation in healthy subjects. Interestingly, the higher FXR1 expression is associated with greater emotional stability, except in the context of higher GSK3 $\beta$ expression (Del'Guidice et al., 2015). Gsk3 $\beta$ is a target of mood stabilizers and may contribute to the action of antipsychotics (Beaulieu et al., 2009). In line with this, animal studies demonstrated that Gsk $3 \beta$ can directly regulate Fxr1 levels in response to mood stabilizers and that increase in Fxr1 expression results in a decrease in anxiety-related behaviors (Del'Guidice et al., 2015; Khlghatyan et al., 2018). An interaction between GSK3B and FXR1 functional polymorphisms was also found in a bipolar patient cohort, where it influenced the severity of manic and depressive symptoms (Bureau et al., 2017).

\section{CONCLUSION}

Overall, several lines of evidence point toward the association of FXR family with neuropsychiatric disorders and its direct or indirect contribution to dopamine receptor signaling. While FXR proteins do not perform redundant functions, they may exert action on common targets like GluA1 and GluA2 via distinct mechanisms. Moreover, engagement of one or the other FXR protein can be a result of different upstream events. Thus, it is possible that FXR proteins may integrate the action of upstream players, such as D1 and D2 dopamine receptors, on common downstream targets, such as GluA1 and GluA2, via distinct mechanisms (Figures 1, 2). Precise investigation of a role of FXR genes has to be carried in animal models in brain region and cell type-specific manner with consideration of developmental stages. Moreover, human functional imaging and genetic studies have to be carried out in context of interaction between FXR genes and their regulators and targets. Investigation of the potential role of FXR family proteins in dopamine signaling may lead to deeper understanding of underpinnings of mental disorders such as schizophrenia and mood disorders and may open new avenues for therapeutic development.

\section{AUTHOR CONTRIBUTIONS}

$\mathrm{JK}$ and J-MB contributed to the redaction of this manuscript.

\section{FUNDING}

J-MB is recipient of a Canada Research Chair Tier 1 in Molecular Psychiatry. This work was funded by a discovery grant from the Canadian Institutes of Health Research (CIHR). 


\section{REFERENCES}

Antar, L. N., Dictenberg, J. B., Plociniak, M., Afroz, R., and Bassell, G. J. (2005). Localization of FMRP-associated mRNA granules and requirement of microtubules for activity-dependent trafficking in hippocampal neurons. Genes Brain Behav. 4, 350-359. doi: 10.1111/j.1601-183X.2005.00128.x

Ashok, A. H., Marques, T. R., Jauhar, S., Nour, M. M., Goodwin, G. M., Young, A. H., et al. (2017). The dopamine hypothesis of bipolar affective disorder: the state of the art and implications for treatment. Mol. Psychiatry 22, 666-679. doi: $10.1038 / \mathrm{mp} .2017 .16$

Bakker, C. E., de Diego Otero, Y., Bontekoe, C., Raghoe, P., Luteijn, T., Hoogeveen, A. T., et al. (2000). Immunocytochemical and biochemical characterization of FMRP, FXR1P, and FXR2P in the mouse. Exp. Cell Res. 258, 162-170. doi: $10.1006 /$ excr.2000.4932

Beaulieu, J. M., and Gainetdinov, R. R. (2011). The physiology, signaling, and pharmacology of dopamine receptors. Pharmacol. Rev. 63, 182-217. doi: 10. 1124/pr.110.002642

Beaulieu, J. M., Gainetdinov, R. R., and Caron, M. G. (2009). Akt/GSK3 signaling in the action of psychotropic drugs. Annu. Rev. Pharmacol. Toxicol. 49, 327-347. doi: 10.1146/annurev.pharmtox.011008.145634

Beaulieu, J. M., Marion, S., Rodriguiz, R. M., Medvedev, I. O., Sotnikova, T. D., Ghisi, V., et al. (2008). A beta-arrestin 2 signaling complex mediates lithium action on behavior. Cell 132, 125-136. doi: 10.1016/j.cell.2007.11.041

Beaulieu, J. M., Sotnikova, T. D., Marion, S., Lefkowitz, R. J., Gainetdinov, R. R., and Caron, M. G. (2005). An Akt/beta-arrestin 2/PP2A signaling complex mediates dopaminergic neurotransmission and behavior. Cell 122, 261-273. doi: 10.1016/j.cell.2005.05.012

Beaulieu, J. M., Sotnikova, T. D., Yao, W. D., Kockeritz, L., Woodgett, J. R., Gainetdinov, R. R., et al. (2004). Lithium antagonizes dopamine-dependent behaviors mediated by an AKT/glycogen synthase kinase 3 signaling cascade. Proc. Natl. Acad. Sci. U.S.A. 101, 5099-5104. doi: 10.1073/pnas.0307921101

Bontekoe, C. J., McIlwain, K. L., Nieuwenhuizen, I. M., Yuva-Paylor, L. A., Nellis, A., Willemsen, R., et al. (2002). Knockout mouse model for Fxr2: a model for mental retardation. Hum. Mol. Genet. 11, 487-498. doi: 10.1093/hmg/ 11.5.487

Bowden, N. A., Scott, R. J., and Tooney, P. A. (2008). Altered gene expression in the superior temporal gyrus in schizophrenia. BMC Genomics 9:199. doi: 10.1186/1471-2164-9-199

Bureau, A., Beaulieu, J. M., Paccalet, T., Chagnon, Y. C., and Maziade, M. (2017). The interaction of GSK3B and FXR1 genotypes may influence the mania and depression dimensions in mood disorders. J. Affect. Disord. 213, 172-177. doi: 10.1016/j.jad.2017.02.023

Caffino, L., Giannotti, G., Malpighi, C., Racagni, G., Filip, M., and Fumagalli, F. (2014). Long-term abstinence from developmental cocaine exposure alters Arc/Arg3.1 modulation in the rat medial prefrontal cortex. Neurotox Res. 26, 299-306. doi: 10.1007/s12640-014-9472-1

Christie, S. B., Akins, M. R., Schwob, J. E., and Fallon, J. R. (2009). The FXG: a presynaptic fragile $\mathrm{X}$ granule expressed in a subset of developing brain circuits. J. Neurosci. 29, 1514-1524. doi: 10.1523/JNEUROSCI.3937-08.2009

Cook, D., Nuro, E., Jones, E. V., Altimimi, H. F., Farmer, W. T., Gandin, V., et al. (2014). FXR1P limits long-term memory, long-lasting synaptic potentiation, and de novo GluA2 translation. Cell Rep. 9, 1402-1416. doi: 10.1016/j.celrep. 2014.10.028

Cook, D., Sanchez-Carbente Mdel, R., Lachance, C., Radzioch, D., Tremblay, S., Khandjian, E. W., et al. (2011). Fragile X related protein 1 clusters with ribosomes and messenger RNAs at a subset of dendritic spines in the mouse hippocampus. PLoS One 6:e26120. doi: 10.1371/journal.pone. 0026120

Crawford, D. C., Acuña, J. M., and Sherman, S. L. (2001). FMR1 and the fragile $\mathrm{X}$ syndrome: human genome epidemiology review. Genet. Med. 3, 359-371. doi: 10.1097/00125817-200109000-00006

Darnell, J. C., Fraser, C. E., Mostovetsky, O., and Darnell, R. B. (2009). Discrimination of common and unique RNA-binding activities among Fragile X mental retardation protein paralogs. Hum. Mol. Genet. 18, 3164-3177. doi: 10.1093/hmg/ddp255

Del' Guidice, T., and Beaulieu, J. M. (2015). Selective disruption of dopamine D2-receptors/beta-arrestin2 signaling by mood stabilizers. J. Recept. Signal Transduct. Res. 35, 224-232. doi: 10.3109/10799893.2015.1072976
Del'Guidice, T., Latapy, C., Rampino, A., Khlghatyan, J., Lemasson, M., Gelao, B., et al. (2015). FXR1P is a GSK3 $\beta$ substrate regulating mood and emotion processing. Proc. Natl. Acad. Sci. U.S.A. 112, E4610-E4619. doi: 10.1073/pnas. 1506491112

Dempster, E. L., Pidsley, R., Schalkwyk, L. C., Owens, S., Georgiades, A., Kane, F., et al. (2011). Disease-associated epigenetic changes in monozygotic twins discordant for schizophrenia and bipolar disorder. Hum. Mol. Genet. 20, 4786-4796. doi: 10.1093/hmg/ddr416

Devor, A., Andreassen, O. A., Wang, Y., Maki-Marttunen, T., Smeland, O. B., Fan, C. C., et al. (2017). Genetic evidence for role of integration of fast and slow neurotransmission in schizophrenia. Mol. Psychiatry 22, 792-801. doi: $10.1038 / \mathrm{mp} .2017 .33$

Du, J., Gray, N. A., Falke, C. A., Chen, W., Yuan, P., Szabo, S. T., et al. (2004). Modulation of synaptic plasticity by antimanic agents: the role of AMPA glutamate receptor subunit 1 synaptic expression. J. Neurosci. 24, 6578-6589. doi: 10.1523/JNEUROSCI.1258-04.2004

El Fatimy, R., Davidovic, L., Tremblay, S., Jaglin, X., Dury, A., Robert, C., et al. (2016). Tracking the fragile $X$ mental retardation protein in a highly ordered neuronal ribonucleoparticles population: a link between stalled polyribosomes and RNA granules. PLoS Genet. 12:e1006192. doi: 10.1371/journal.pgen. 1006192

Fatemi, S. H., and Folsom, T. D. (2011). The role of fragile X mental retardation protein in major mental disorders. Neuropharmacology 60, 1221-1226. doi: 10.1016/j.neuropharm.2010.11.011

Folsom, T. D., Thuras, P. D., and Fatemi, S. H. (2015). Protein expression of targets of the FMRP regulon is altered in brains of subjects with schizophrenia and mood disorders. Schizophr. Res. 165, 201-211. doi: 10.1016/j.schres.2015. 04.012

Fromer, M., Pocklington, A. J., Kavanagh, D. H., Williams, H. J., Dwyer, S., Gormley, P., et al. (2014). De novo mutations in schizophrenia implicate synaptic networks. Nature 506, 179-184. doi: 10.1038/nature 12929

Gainetdinov, R. R., Bohn, L. M., Sotnikova, T. D., Cyr, M., Laakso, A., Macrae, A. D., et al. (2003). Dopaminergic supersensitivity in G protein-coupled receptor kinase 6-deficient mice. Neuron 38, 291-303. doi: 10.1016/S08966273(03)00192-2

Goes, F. S., Pirooznia, M., Parla, J. S., Kramer, M., Ghiban, E., Mavruk, S., et al. (2016). Exome Sequencing of Familial Bipolar Disorder. JAMA Psychiatry 73, 590-597. doi: 10.1001/jamapsychiatry.2016. 0251

Guo, W., Polich, E. D., Su, J., Gao, Y., Christopher, D. M., Allan, A. M., et al. (2015). Fragile X proteins FMRP and FXR2P control synaptic GluA1 expression and neuronal maturation via distinct mechanisms. Cell Rep. 11, 1651-1666. doi: 10.1016/j.celrep.2015.05.013

Gurevich, E. V., Gainetdinov, R. R., and Gurevich, V. V. (2016). G protein-coupled receptor kinases as regulators of dopamine receptor functions. Pharmacol. Res. 111, 1-16. doi: 10.1016/j.phrs.2016.05.010

Hauberg, M. E., Roussos, P., Grove, J., Børglum, A. D., Mattheisen, M., and Schizophrenia Working Group of the Psychiatric Genomics Consortium (2016). Analyzing the role of microRNAs in schizophrenia in the context of common genetic risk variants. JAMA Psychiatry 73, 369-377. doi: 10.1001/ jamapsychiatry.2015.3018

Howes, O. D., and Kapur, S. (2009). The dopamine hypothesis of schizophrenia: version III-the final common pathway. Schizophr. Bull. 35, 549-562. doi: 10. 1093/schbul/sbp006

Khlghatyan, J., Evstratova, A., Chamberland, S., Marakhovskaia, A., Bahremand, A., Toth, K., et al. (2018). Fxrl and its negative regulator Gsk3 $\beta$ are modulators of anxiety and glutamatergic neurotransmission. Front. Mol. Neurosci. 11:119. doi: 10.3389/fnmol.2018. 00119

Kovács, T., Kelemen, O., and Kéri, S. (2013). Decreased fragile X mental retardation protein (FMRP) is associated with lower IQ and earlier illness onset in patients with schizophrenia. Psychiatry Res. 210, 690-693. doi: 10.1016/j.psychres.2012. 12.022

Kumar, V., Kim, K., Joseph, C., Kourrich, S., Yoo, S. H., Huang, H. C., et al. (2013). C57BL/6N mutation in cytoplasmic FMRP interacting protein 2 regulates cocaine response. Science 342, 1508-1512. doi: 10.1126/science. 1245503 
Latapy, C., Rioux, V., Guitton, M. J., and Beaulieu, J. M. (2012). Selective deletion of forebrain glycogen synthase kinase 3 beta reveals a central role in serotoninsensitive anxiety and social behaviour. Philos. Trans. R. Soc. Lond. B Biol. Sci. 367, 2460-2474. doi: 10.1098/rstb.2012.0094

Liedtke, E., Zhang, S., Thompson, J., Sillau, S., and Gault, J. (2017). Correlated expression analysis of genes implicated in schizophrenia: identification of putative disease-related pathways. New Horiz. Transl. Med. 3, 224-232.

Liu, X., Kelsoe, J. R., Greenwood, T. A., and Bipolar Genome Study [BIGS] (2016). A genome-wide association study of bipolar disorder with comorbid eating disorder replicates the SOX2-OT region. J. Affect. Disord. 189, 141-149. doi: 10.1016/j.jad.2015.09.029

Mientjes, E. J., Willemsen, R., Kirkpatrick, L. L., Nieuwenhuizen, I. M., HoogeveenWesterveld, M., Verweij, M., et al. (2004). Fxrl knockout mice show a striated muscle phenotype: implications for Fxrlp function in vivo. Hum. Mol. Genet. 13, 1291-1302. doi: $10.1093 / \mathrm{hmg} / \mathrm{ddh} 150$

Mines, M. A., and Jope, R. S. (2011). Glycogen synthase kinase-3: a promising therapeutic target for fragile x syndrome. Front. Mol. Neurosci. 4:35. doi: 10. 3389/fnmol.2011.00035

Missale, C., Nash, S. R., Robinson, S. W., Jaber, M., and Caron, M. G. (1998). Dopamine receptors: from structure to function. Physiol. Rev. 78, 189-225. doi: 10.1152/physrev.1998.78.1.189

Purcell, S. M., Moran, J. L., Fromer, M., Ruderfer, D., Solovieff, N., Roussos, P., et al. (2014). A polygenic burden of rare disruptive mutations in schizophrenia. Nature 506, 185-190. doi: 10.1038/nature12975

Renthal, W., Carle, T. L., Maze, I., Covington, H. E. III, Truong, H. T., Alibhai, I., et al. (2008). Delta FosB mediates epigenetic desensitization of the c-fos gene after chronic amphetamine exposure. J. Neurosci. 28, 7344-7349. doi: 10.1523/ JNEUROSCI.1043-08.2008

Renthal, W., and Nestler, E. J. (2008). Epigenetic mechanisms in drug addiction. Trends Mol. Med. 14, 341-350. doi: 10.1016/j.molmed.2008.06.004

Schizophrenia Working Group of the Psychiatric Genomics Consortium (2014). Biological insights from 108 schizophrenia-associated genetic loci. Nature 511, 421-427. doi: 10.1074/jbc.M113.535203

Shi, S., Leites, C., He, D., Schwartz, D., Moy, W., Shi, J., et al. (2014). MicroRNA9 and microRNA-326 regulate human dopamine D2 receptor expression, and the microRNA-mediated expression regulation is altered by a genetic variant. J. Biol. Chem. 289, 13434-13444. doi: 10.1074/jbc.M113.535203

Smith, L. N., Jedynak, J. P., Fontenot, M. R., Hale, C. F., Dietz, K. C., Taniguchi, M., et al. (2014). Fragile X mental retardation protein regulates synaptic and behavioral plasticity to repeated cocaine administration. Neuron 82, 645-658. doi: 10.1016/j.neuron.2014.03.028

Snyder, G. L., Allen, P. B., Fienberg, A. A., Valle, C. G., Huganir, R. L., Nairn, A. C., et al. (2000). Regulation of phosphorylation of the GluR1 AMPA receptor in the neostriatum by dopamine and psychostimulants in vivo. J. Neurosci. 20, 4480-4488. doi: 10.1093/hmg/ddl121

Spencer, C. M., Serysheva, E., Yuva-Paylor, L. A., Oostra, B. A., Nelson, D. L., and Paylor, R. (2006). Exaggerated behavioral phenotypes in Fmr1/Fxr2 double knockout mice reveal a functional genetic interaction between Fragile X-related proteins. Hum. Mol. Genet. 15, 1984-1994. doi: 10.15252/emmm.201 505696

Stepniak, B., Kästner, A., Poggi, G., Mitjans, M., Begemann, M., Hartmann, A., et al. (2015). Accumulated common variants in the broader fragile X gene family modulate autistic phenotypes. EMBO Mol. Med. 7, 1565-1579. doi: 10.15252/ emmm.201505696

Svenningsson, P., Nishi, A., Fisone, G., Girault, J. A., Nairn, A. C., and Greengard, P. (2004). DARPP-32: an integrator of neurotransmission. Annu. Rev. Pharmacol. Toxicol. 44, 269-296. doi: 10.1038/ncomms14519
Takata, A., Matsumoto, N., and Kato, T. (2017). Genome-wide identification of splicing QTLs in the human brain and their enrichment among schizophreniaassociated loci. Nat. Commun. 8:14519. doi: 10.1038/ncomms14519

Tamanini, F., Willemsen, R., van Unen, L., Bontekoe, C., Galjaard, H., Oostra, B. A., et al. (1997). Differential expression of FMR1, FXR1 and FXR2 proteins in human brain and testis. Hum. Mol. Genet. 6, 1315-1322. doi: 10.1016/j.celrep. 2017.06.038

Thomas, K. T., Anderson, B. R., Shah, N., Zimmer, S. E., Hawkins, D., Valdez, A. N., et al. (2017). Inhibition of the schizophrenia-associated microRNA miR137 disrupts $\mathrm{Nrg} 1 \alpha$ neurodevelopmental signal transduction. Cell Rep. 20, 1-12. doi: 10.1016/j.celrep.2017.06.038

Thomsen, R., Pallesen, J., Daugaard, T. F., Børglum, A. D., and Nielsen, A. L. (2013). Genome wide assessment of mRNA in astrocyte protrusions by direct RNA sequencing reveals mRNA localization for the intermediate filament protein nestin. Glia 61, 1922-1937. doi: 10.1002/glia.22569

Ventura, R., Pascucci, T., Catania, M. V., Musumeci, S. A., and Puglisi-Allegra, S. (2004). Object recognition impairment in Fmrl knockout mice is reversed by amphetamine: involvement of dopamine in the medial prefrontal cortex. Behav. Pharmacol. 15, 433-442. doi: 10.1074/jbc.M110.116293

Wang, H., Kim, S. S., and Zhuo, M. (2010). Roles of fragile X mental retardation protein in dopaminergic stimulation-induced synapse-associated protein synthesis and subsequent alpha-amino-3-hydroxyl-5-methyl-4-isoxazole-4propionate (AMPA) receptor internalization. J. Biol. Chem. 285, 21888-21901. doi: $10.1074 /$ jbc.M110.116293

Wang, H., Wu, L. J., Kim, S. S., Lee, F. J., Gong, B., Toyoda, H., et al. (2008). FMRP acts as a key messenger for dopamine modulation in the forebrain. Neuron 59 , 634-647. doi: 10.1016/j.neuron.2008.06.027

Xu, X. L., Zong, R., Li, Z., Biswas, M. H., Fang, Z., Nelson, D. L., et al. (2011). FXR1P but not FMRP regulates the levels of mammalian brain-specific microRNA-9 and microRNA-124. J. Neurosci. 31, 13705-13709. doi: 10.1523/JNEUROSCI. 2827-11.2011

Yao, W. D., Gainetdinov, R. R., Arbuckle, M. I., Sotnikova, T. D., Cyr, M., Beaulieu, J. M., et al. (2004). Identification of PSD-95 as a regulator of dopaminemediated synaptic and behavioral plasticity. Neuron 41, 625-638. doi: 10.1016/ S0896-6273(04)00048-0

Yuskaitis, C. J., Mines, M. A., King, M. K., Sweatt, J. D., Miller, C. A., and Jope, R. S. (2010). Lithium ameliorates altered glycogen synthase kinase-3 and behavior in a mouse model of fragile X syndrome. Biochem. Pharmacol. 79, 632-646. doi: 10.1016/j.bcp.2009.09.023

Zhang, J., Hou, L., Klann, E., and Nelson, D. L. (2009). Altered hippocampal synaptic plasticity in the FMR1 gene family knockout mouse models. J. Neurophysiol. 101, 2572-2580. doi: 10.1152/jn.90558.2008

Zhang, Y., Chen, K., Sloan, S. A., Bennett, M. L., Scholze, A. R., O'Keeffe, S., et al. (2014). An RNA-sequencing transcriptome and splicing database of glia, neurons, and vascular cells of the cerebral cortex. J. Neurosci. 34, 11929-11947. doi: 10.1523/JNEUROSCI.1860-14.2014

Conflict of Interest Statement: The authors declare that the research was conducted in the absence of any commercial or financial relationships that could be construed as a potential conflict of interest.

Copyright $\odot 2018$ Khlghatyan and Beaulieu. This is an open-access article distributed under the terms of the Creative Commons Attribution License (CC BY). The use, distribution or reproduction in other forums is permitted, provided the original author(s) and the copyright owner(s) are credited and that the original publication in this journal is cited, in accordance with accepted academic practice. No use, distribution or reproduction is permitted which does not comply with these terms. 